\title{
Impact of Mulch, Cover Crop and Herbicides on Growth, Yield and Nutrient Uptake in Direct Seeded Rice (Oryza sativa L.)
}

Pratik Sanodiya, Manoj Kumar Singh

10.18805/ag.D-5506

\begin{abstract}
Background: Direct seeded rice is very much prone for weed infestation in eastern Uttar Pradesh. The aim of this trial is to find out appropriate option for improving rice growth and yields besides increasing nutrient uptake.

Methods: A field investigation was conducted during the Kharif season of 2014 and 2015 at Varanasi, Uttar Pradesh. Rice variety MTU-7029 was sown with Sesbania and sunhemp cover crop bispyribac Na $25 \mathrm{~g}$ ha $^{-1}+$ azimsulfuron $30 \mathrm{~g}^{-1}$ at $15 \mathrm{DAS}$ and $2,4-\mathrm{D} 0.5$ $\mathrm{kg} \mathrm{ha}^{-1}$ at 30 DAS were applied to find out best weed control practice for enhancing growth, yields and nutrient uptake in direct seeded rice. Result: Sesbania cover crop fb bispyribac $25 \mathrm{~g} \mathrm{ha}^{-1}+$ azimsulfuron $30 \mathrm{~g} \mathrm{ha}^{-1}$ at 15 DAS markedly improved plant height, dry matter

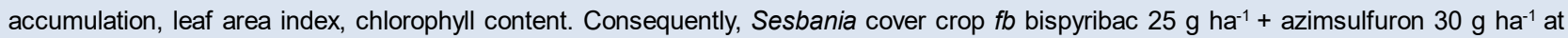
15 DAS influenced statistically grain and straw yields. Sesbania cover crop fb bispyribac $25 \mathrm{~g} \mathrm{ha}^{-1}+$ azimsulfuron $30 \mathrm{~g}$ ha-1 at $15 \mathrm{DAS}^{-1}$ recorded significantly lower nutrients (NPK and $\mathrm{Zn}$ ) depletion by weeds and higher nutrients (NPK and $\mathrm{Zn}$ ) uptake as compared to hand weeding at 15 and 35 DAS in rice.
\end{abstract}

Key words: Azimsulfuron, Bispyribac Na, Direct seeded rice, Sesbania, Sunhemp.

\section{INTRODUCTION}

India stand second in rice (Oryza sativa L.) production (163 $\mathrm{Mt})$ after China, while first in its import (13 Mt) and $42^{\text {nd }}$ in average yield (3.76 t ha-1), indicating very low rice yield per hectare (Sanodiya and Singh (2019). Rice is grown under diverse ecologies ranging from irrigated to rainfed upland, lowland and deep water. Direct seeded rice is becoming more popular as an alternative to transplanted rice, as it is more remunerative if the crop managed properly (Sharma et al., 2007). Aerobic edaphic conditions under non flooded conditions in DSR stimulate germination of diverse weed species. Sunil et al. (2010) reported that season-long weed competition in direct-seeded rice may cause yield reduction up to $80 \%$. Raj et al. (2013) found $72 \%$ reduction in grain yield due to the infestation of non-grassy, broad-leaved weeds and sedges in direct-seeded rice. Growing cover crops, have potential as an important component of a system oriented ecological weed management strategy for sustainable agriculture (Kruidhof et al., 2008), because it conserve soil and moisture, enhancing soil nutrient status (Malviya and Singh, 2007), total biomass production. The inclusion of cover crops in the rotation at a time when land might otherwise lie uncropped will suppress weed development, yet maintain soil fertility and prevent erosion (Liebman and Davis, 2000). Sesbania cover crop followed by (fb) bispyribac $25 \mathrm{~g} / \mathrm{ha}+$ azimsulfuron $30 \mathrm{~g} / \mathrm{ha}$ at $15 \mathrm{DAS}$ reduced weed density of various weeds and dry weight than sunnhemp (Crotalaria juncea L.) cover crop fb bispyribac $25 \mathrm{~g} / \mathrm{ha}+$ azimsulfuron $30 \mathrm{~g} / \mathrm{ha}$ at 15 DAS thus resulting in the lowest weed index except hand weeding at 15 and 35 DAS (Sanodiya and Singh, 2018). Through allelopathic (Kruidhof et al., 2008b) and mechanical effects (Den Hollander et al., 2007a) and the competition between the
Department of Agronomy, Institute of Agricultural Sciences, Banaras Hindu University, Varanasi-221 005, Uttar Pradesh, India.

Corresponding Author: Pratik Sanodiya, Department of Agronomy, Institute of Agricultural Sciences, Banaras Hindu University, Varanasi-221 005, Uttar Pradesh, India.

Email: prsanodiya10@gmail.com

How to cite this article: Sanodiya, P. and Singh, M.K. (2022). Impact of Mulch, Cover Crop and Herbicides on Growth, Yield and Nutrient Uptake in Direct Seeded Rice (Oryza sativa L.). Agricultural Science Digest. DOI: 10.18805/ag.D-5506.

Submitted: 05-10-2021 Accepted: 28-12-2021 Online: 17-02-2022

cover crops and the weeds for limited resources such as light, water and nutrients (Kruidhof et al., 2008a). According to Singh et al., (2006) amongst the weed control treatments, broadcasting of Sesbania knocked down by the application of 2, 4-D at 30 days after sowing recorded lowest weed dry weight. Co-culture of Sesbaniain rice and its subsequent knock down by 2, 4-D ester reduced the weed population by nearly half without any adverse effect on rice yield (Gupta et al., 2006). Weed problem in direct seeded rice can be managed in Gangetic plains by integrated various control measures. However, weeds in direct seeded rice cannot be controlled by incorporation of cover crops and live mulches alone because of various flushes of weeds during crop growth. It is imperative to identify effective integrated weed management practices for minimizing nutrient uptake by weeds and maximizing by crop yield and nutrient uptake. Therefore, keeping these points in view, the present study was taken up, to assess the efficacy of different herbicides along with growing cover crops and live mulches and to study the impact of integrated weed management on 
Impact of Mulch, Cover Crop and Herbicides on Growth, Yield and Nutrient Uptake in Direct Seeded Rice (Oryza sativa L.)

growth, yield and nutrient uptake by weeds and crop in direct seeded rice.

\section{METHODS AND MATERIALS}

A field experiment was conducted during Kharif season of 2014 and 2015 at Agricultural Research Farm, Institute of Agricultural Sciences, Banaras Hindu University, Varanasi, Uttar Pradesh. The soil was sandy clay loam, with $\mathrm{pH} 7.40$, low in available organic carbon $(0.41 \%)$, available nitrogen (207.47 kg/ha) and medium in available phosphorous (23.85 $\mathrm{kg} / \mathrm{ha})$ and potassium $(219.60 \mathrm{~kg} / \mathrm{ha})$. The experiment was laid out in a randomized block design, compried of treatments, viz. Sesbania cover crop fb bispyribac $25 \mathrm{~g} \mathrm{ha}^{-1}$ + azimsulfuron 30 gha $^{-1}$ at 15 DAS, sunhemp cover crop fb bispyribac $25 \mathrm{~g} \mathrm{ha}^{-1}+$ azimsulfuron $30 \mathrm{~g} \mathrm{ha}^{-1}$ at 15 DAS, Sesbania cover crop fb Sesbania coculture $\mathrm{fb} 2,4 \mathrm{D} 0.5 \mathrm{~kg}$ $\mathrm{ha}^{-1}$ at 30 DAS, sunhemp cover crop fb sunhemp coculture fb 2, 4 D $0.5 \mathrm{~kg} \mathrm{ha}^{-1}$ at 30 DAS, Sesbania coculture fb 2, 4 D $0.5 \mathrm{~kg} \mathrm{ha}^{-1}$ at 30 DAS, sunhemp coculture fb 2, 4- D 0.5 $\mathrm{kg} \mathrm{ha}^{-1}$ at 30 DAS, bispyribac Na $25 \mathrm{~g} \mathrm{ha}^{-1}+$ azimsulfuron 30 $\mathrm{g} \mathrm{ha}^{-1}$ at $15 \mathrm{DAS}$, hand weeding at 15 and 35 DAS and weedy check during both the years and replicated thrice. Rice variety MTU-7029 was sown by zero till drill during the last week of June in the both the years following the seed rate of $30 \mathrm{~kg} / \mathrm{ha}$ and $20 \mathrm{~cm}$ row-row spacing. A recommended dose of fertilizer ( $150 \mathrm{~kg} \mathrm{~N}, 60 \mathrm{~kg} \mathrm{P}_{2} \mathrm{O}_{5}$ and $60 \mathrm{~kg} \mathrm{~K} \mathrm{O}_{2}$ ) was applied through inorganic sources viz urea, single super phosphate and muriate of potash during both the years of experimentation. Full dose of phosphorus and potash were applied as basal application while nitrogen was applied half as basal and remaining half at two equal parts at tillering and panicle initiation stage of rice. Application of alone and tank mixed post emergence herbicides was done according to the treatments using knap-sack sprayer fitted with flatfan nozzle. The spray volume of post emergence herbicides was 600 litres/ha. The crop was raised under irrigated condition under the recommended package of practices. Five plants from each experimental plot were selected randomly and tagged. Plant height $(\mathrm{cm})$ of the rice was recorded with the help of meter scale from base of the plant to the tip of upper most leaf of the plant before panicle emergence and upto the tip of panicle after heading, then averaged and expressed in $\mathrm{cm}$. The leaf area index (LAI) is the area of leaf surface per unit area of land surface. Leaf area index was measured by using portable plant canopy analyzer at five randomly selected sites in each plot at 90 DAS. Dry matter accumulation was measured from two randomly selected places in plot and then cut one plant row near ground level of one meter length from each plot at different stages of crop growth from either side leaving two rows. Samples were first dried in sun and then oven dried at $65^{\circ} \mathrm{C}$ till the constant weight was achieved. After drying, the samples were weighed for recording dry weight and expressed in $\mathrm{g} / \mathrm{mrow}$ length. The data was analyzed statistically using Duncan multiple range test (DMRT) for valid conclusion of research.
Nutrient uptake by grain and straw of rice crop was calculated by multiplying yields of grain or straw with its nutrient contents (Black et al. 1965).

Nutrient uptake $\left(\mathrm{kg} \mathrm{ha}^{-1}\right)=$

$$
\frac{\text { Nutrient content }(\%) \text { in grain or straw }}{\text { Grain/Straw yield }\left(\mathrm{kg} \mathrm{ha}^{-1}\right)} \times 100
$$

Nutrient (N, P, K and $\mathrm{Zn}$ ) removal by weeds ( $\mathrm{kg} \mathrm{ha}^{-1}$ and $\mathrm{g} \mathrm{ha}^{-1}$ ) Nutrient (N, P, K and $\mathrm{Zn}$ ) removal by weeds was calculated as per the following formula.

Nutrient removal by weeds $\left(\mathrm{kg} \mathrm{ha}^{-1}\right)=$

$$
\frac{\text { Nutrient content }(\%) \text { in weed }}{\text { Weed dry matter }\left(\mathrm{kg} \mathrm{ha}^{-1}\right)} \times 100
$$

\section{RESULTS AND DISCUSSION}

\section{Growth attributes}

Amongst the integrated weed management treatments, Sesbania cover crop fb bispyribac $25 \mathrm{~g} \mathrm{ha}^{-1}+$ azimsulfuron $30 \mathrm{~g} \mathrm{ha}^{-1}$ at 15 DAS resulted higher plant height, dry matter accumulation $(\mathrm{g} / \mathrm{m})$, leaf area index and chlorophyll content at 90 DAS in comparison to sunhemp cover crop fb bispyribac $25 \mathrm{~g} \mathrm{ha}^{-1}+$ azimsulfuron $30 \mathrm{~g} \mathrm{ha}^{-1}$ at $15 \mathrm{DAS}$ and both treatments were statistically similar to each other during field experimentation years (Table 1). However, bispyribac $25 \mathrm{~g} \mathrm{ha}^{-1}+$ azimsulfuron $30 \mathrm{~g} \mathrm{ha}^{-1}$ at 15 DAS recorded significantly higher plant height, dry matter accumulation $(\mathrm{g} / \mathrm{m})$, leaf area index and chlorophyll content at 90 DAS as compared to Sesbania cover crop fb Sesbania coculture $f b$ 2,-4 D $0.5 \mathrm{~kg} \mathrm{ha}^{-1}$ at 30 DAS, sunhemp cover crop $\mathrm{fb}$ sunhemp coculture $\mathrm{fb} 2,4 \mathrm{D} 0.5 \mathrm{~kg} \mathrm{ha}^{-1}$ at $30 \mathrm{DAS}$, Sesbania coculture $\mathrm{fb} 2,4-\mathrm{D} 0.5 \mathrm{~kg} \mathrm{ha}^{-1}$ at 30 DAS and sunhemp coculture fb 2, 4 D $0.5 \mathrm{~kg} \mathrm{ha}^{-1}$ at 30 DAS (Table 1). Majhi et al, (2009) also expressed similar views in direct seeded rice.Amongst the integrated weed management treatments, Sesbania cover crop fb bispyribac $25 \mathrm{~g} \mathrm{ha}^{-1}+$ azimsulfuron $30 \mathrm{~g} \mathrm{ha}^{-1}$ at 15 DAS recorded higher plant height, dry matter accumulation $\mathrm{g} / \mathrm{m}, \mathrm{LAl}$ and chlorophyll content at $90 \mathrm{DAS}$ in comparison to sunhemp cover crop fb bispyribac $25 \mathrm{~g} \mathrm{ha}^{-1}+$ azimsulfuron $30 \mathrm{~g} \mathrm{ha}^{-1}$ at 15 DAS. This could be attributed to higher weed control efficiency under these treatments as a result of which crop confronted minimum competition from weeds for growth factors like moisture, nutrient, light and space. The weeds were controlled at initial stage due to cover crops. Mechanical weeding to kill the cover crop and leave a thick evenly distributed layer of weed suppressing mulch. This surface mulch may limit further weed development through its effect on light transmittance, soil temperature and soil moisture. The mulch also provides a protective habitat for seed predators which helps to reduce viz. dry matter accumulation finally weed seed numbers. These resultsalso supported by Majhi et al., (2009), Reader, (1991) and Teasdale, (1993). The tank mixed application of bispyribac $25 \mathrm{~g} \mathrm{ha}^{-1}+$ azimsulfuron $30 \mathrm{~g} \mathrm{ha}^{-1}$ at 15 DAS was found effective to control the second flush of weeds. Similar findings were recorded by Singh et al., (2014). 
Table 1: Effect of integrated weed management on growth attributes at 90 DAS and yields of direct seeded rice (average of 2 years).

\begin{tabular}{|c|c|c|c|c|c|c|}
\hline Treatment & $\begin{array}{l}\text { Plant } \\
\text { height } \\
(\mathrm{cm})\end{array}$ & $\begin{array}{l}\text { Dry matter } \\
\text { accumulation } \\
\quad\left(\mathrm{gm}^{-1}\right)\end{array}$ & $\begin{array}{l}\text { LAI (Leaf } \\
\text { area } \\
\text { index) }\end{array}$ & $\begin{array}{c}\text { Chlorophyll } \\
\text { content } \\
\text { (SPAD value) }\end{array}$ & $\begin{array}{l}\text { Grain } \\
\text { yield } \\
\left(\mathrm{t} \mathrm{ha}^{-1}\right)\end{array}$ & $\begin{array}{c}\text { Straw } \\
\text { yield } \\
\left(\mathrm{t} \mathrm{ha}^{-1}\right)\end{array}$ \\
\hline $\begin{aligned} \mathrm{T}_{1}: & \text { Sesbania cover crop fb bispyribac } 25 \mathrm{~g} \mathrm{ha}^{-1}+ \\
& \text { azimsulfuron } 30 \mathrm{~g} \mathrm{ha}^{-1} \text { at } 15 \text { DAS }\end{aligned}$ & 87.33 & 111.25 & 3.86 & 43.61 & 48.31 & 59.91 \\
\hline $\begin{array}{l}\mathrm{T}_{2}: \text { Sunhemp cover crop fb bispyribac } 25 \mathrm{~g} \mathrm{ha}^{-1}+ \\
\text { azimsulfuron } 30 \mathrm{~g} \mathrm{ha}^{-1} \text { at } 15 \mathrm{DAS}\end{array}$ & 87.12 & 110.63 & 3.85 & 42.92 & 47.62 & 57.79 \\
\hline $\begin{array}{l}\mathrm{T}_{3}: \text { Sesbania cover crop fb Sesbania coculture fb } 2 \text {, } \\
4 \mathrm{D} 0.5 \mathrm{~kg} \mathrm{ha}^{-1} \text { at } 30 \mathrm{DAS}\end{array}$ & 85.51 & 109.97 & 3.83 & 42.21 & 46.52 & 57.05 \\
\hline $\begin{array}{l}T_{4}: \text { Sunhemp cover crop fb Sunhemp coculture } f b 2 \text {, } \\
\text { 4 D } 0.5 \mathrm{~kg} \mathrm{ha}^{-1} \text { at } 30 \text { DAS }\end{array}$ & 85.18 & 109.37 & 3.82 & 42.12 & 45.96 & 56.83 \\
\hline $\mathrm{T}_{5}$ : Sesbania coculture $\mathrm{fb} 2,4$-D $0.5 \mathrm{~kg} \mathrm{ha}^{-1}$ at $30 \mathrm{DAS}$ & 85.09 & 108.95 & 3.81 & 41.86 & 45.50 & 56.01 \\
\hline $\mathrm{T}_{6}:$ Sunhemp coculture $\mathrm{fb} 2,4-\mathrm{D} 0.5 \mathrm{~kg} \mathrm{ha}^{-1}$ at 30 DAS & 84.78 & 108.61 & 3.80 & 41.71 & 45.03 & 55.47 \\
\hline $\mathrm{T}_{7}$ : Bispyribac $25 \mathrm{~g} \mathrm{ha}^{-1}+$ azimsulfuron $30 \mathrm{~g} \mathrm{ha}^{-1}$ at 15 DAS & 86.05 & 110.35 & 3.84 & 42.59 & 47.07 & 57.83 \\
\hline $\mathrm{T}_{8}:$ Hand weeding at 15 and 35 DAS & 89.78 & 117.19 & 4.04 & 44.50 & 57.47 & 70.88 \\
\hline $\mathrm{T}_{9}:$ Weedy & 69.04 & 75.20 & 2.02 & 36.80 & 23.52 & 33.67 \\
\hline $\mathrm{SEm} \pm$ & 0.90 & 0.87 & 0.06 & 0.12 & 0.08 & 2.02 \\
\hline CD $(5 \%)$ & $2.70^{*}$ & $2.60^{*}$ & $0.18^{*}$ & $0.42^{*}$ & $2.41^{*}$ & $6.06^{*}$ \\
\hline
\end{tabular}

${ }^{*}$ Significant at $P \leq 0.05$; NS- Non significant at $P>0.05$.

\section{Grain and straw yields}

The increase in grain yield under Sesbania cover crop $f b$ bispyribac $25 \mathrm{~g} \mathrm{ha}^{-1}+$ azimsulfuron $30 \mathrm{~g} \mathrm{ha}^{-1}$ at 15 DAS and sunhemp cover crop fb bispyribac $25 \mathrm{~g} \mathrm{ha}^{-1}+$ azimsulfuron $30 \mathrm{~g} \mathrm{ha}^{-1}$ at 15 DAS was $105.39 \%$ and $102.46 \%$ over weedy. Similarly, $77.93 \%$ and $71.63 \%$ higher straw yield under treatments Sesbania cover crop fb bispyribac $25 \mathrm{~g} \mathrm{ha}^{-1}+$ azimsulfuron $30 \mathrm{~g} \mathrm{ha}^{-1}$ at 15 DAS and sunhemp cover crop fb bispyribac $25 \mathrm{~g} \mathrm{ha}^{-1}+$ azimsulfuron $30 \mathrm{~g} \mathrm{ha}^{-1}$ at 15 DAS over weedy, respectively, were recorded. These findings are confronted by Sanodiya and Singh (2017) who reported that Sesbania cover crop fb bispyribac $25 \mathrm{~g} / \mathrm{ha}+$ azimsulfuron $30 \mathrm{~g} / \mathrm{ha}$ at 15 DAS improved grain and straw yields during both years but higher harvest index in 2014 as compared to sunhemp cover crop fb bispyribac Na $25 \mathrm{~g} / \mathrm{ha}+$ azimsulfuron $30 \mathrm{~g} / \mathrm{ha}$ at $15 \mathrm{DAS}$. This was due to Sesbania might have supplied sufficient nutrients in soil after decomposition coupled with smothering effect on weeds during initial stages which resulted in increased growth and yield attributes. Similar findings were also reported by Majhi et al., (2009). These treatments provided almost weed free condition during period of crop-weed competition. The favourable effect of these treatments allowed the crop plants to utilize the moisture, nutrient, light and space more efficiently, resulting in to better growth and yield attributes as compared to other integrated weed management treatments. This result could be attributed to higher weed control efficiency and lower weed index, increased dry matter accumulation and improved yield attributing characters. The minimum grain and straw yields were recorded under weedy due to more weed infestation and their dry matter accumulation and lower values of yield attributing characters. These results are in accordance with findings of Chongtham et al., (2016).

\section{Available nutrients in soil}

After harvesting of crop, soil was analyzed for available soil nutrient and data was analyzed and tabulated in (Table 2). Amongst integrated weed management treatments, Sesbania cover crop fb bispyribac $25 \mathrm{~g} \mathrm{ha}^{-1}+$ azimsulfuron $30 \mathrm{~g} \mathrm{ha}^{-1}$ at 15 DAS recorded significantly higher available nutrients (NPK) in comparison to sunhemp cover crop fb bispyribac $25 \mathrm{~g} \mathrm{ha}^{-1}+$ azimsulfuron $30 \mathrm{~g} \mathrm{ha}^{-1}$ at $15 \mathrm{DAS}$, Sesbania cover crop fb Sesbania coculture fb 2, 4 D $0.5 \mathrm{~kg}$ $\mathrm{ha}^{-1}$ at 30 DAS, sunhemp cover crop fb sunhemp coculture fb 2, 4 D $0.5 \mathrm{~kg} \mathrm{ha}^{-1}$ at 30 DAS, Sesbania coculture fb 2, 4 D $0.5 \mathrm{~kg} \mathrm{ha}^{-1}$ at 30 DAS, sunhemp coculture $\mathrm{fb} 2,4-\mathrm{D} 0.5$ $\mathrm{kg} \mathrm{ha}^{-1}$ at 30 DAS, bispyribac $25 \mathrm{~g} \mathrm{ha}^{-1}+$ azimsulfuron $30 \mathrm{~g}$ $\mathrm{ha}^{-1}$ at 15 DAS (Table 2). Similar findings reported by Sanodiya and Singh (2018) that repeated hand weedings in direct seeded rice recorded minimum nitrogen losses due to weeds. Amongst integrated weed management treatments, Sesbania cover crop fb bispyribac $25 \mathrm{gha}^{-1}+$ azimsulfuron $30 \mathrm{~g} \mathrm{ha}^{-1}$ at 15 DAS recorded significantly higher available nutrients (NPK) in comparison to sunhemp cover crop fb bispyribac $25 \mathrm{~g} \mathrm{ha}^{-1}+$ azimsulfuron $30 \mathrm{~g} \mathrm{ha}^{-1}$ at 15 DAS, Sesbania cover crop fb Sesbania coculture fb 2, 4 D $0.5 \mathrm{~kg} \mathrm{ha}^{-1}$ at 30 DAS, sunhemp cover crop fb sunhemp coculture fb 2, 4 D $0.5 \mathrm{~kg} \mathrm{ha}^{-1}$ at 30 DAS, Sesbania coculture fb 2, 4 -D $0.5 \mathrm{~kg} \mathrm{ha}^{-1}$ at 30 DAS, sunhempcoculture fb 2, 4D $0.5 \mathrm{~kg} \mathrm{ha}^{-1}$ at 30 DAS, bispyribac Na $25 \mathrm{gha}^{-1}+$ azimsulfuron $30 \mathrm{~g} \mathrm{ha}^{-1}$ at $15 \mathrm{DAS}$. This could be found due to less germination of weeds occurred in cover crop treated at initial growth of rice followed by bispyribac $\mathrm{Na}+$ azimsulfuron at later stage. Coculture technology followed by 2, 4-D reduced weed growth and less uptake by weeds. Similar findings also reported by Sanodiya and Singh (2018) that repeated hand weedings in direct seeded rice recorded minimum nitrogen losses due to weeds. 


\section{Nutrient depletion by weeds}

Amongst the integrated weed management treatments, Sesbania cover crop fb bispyribac $25 \mathrm{~g} \mathrm{ha}^{-1}+$ azimsulfuron $30 \mathrm{~g} \mathrm{ha}^{-1}$ at 15 DAS recorded significantly lesser nutrients (NPKZn) depletion by weeds as compared to sunhemp cover crop fb bispyribac $25 \mathrm{~g} \mathrm{ha}^{-1}+$ azimsulfuron $30 \mathrm{~g} \mathrm{ha}^{-1}$ at 15 DAS, Sesbania cover crop fb Sesbania coculture $f b 2,4$ D $0.5 \mathrm{~kg} \mathrm{ha}^{-1}$ at 30 DAS, sunhemp cover crop $\mathrm{fb}$ sunhempcoculture fb 2, 4 -D $0.5 \mathrm{~kg} \mathrm{ha}^{-1}$ at 30 DAS, Sesbania coculture fb 2, 4 -D $0.5 \mathrm{~kg} \mathrm{ha}^{-1}$ at 30 DAS, sunhemp coculture fb 2, 4- D $0.5 \mathrm{~kg} \mathrm{ha}^{-1}$ at 30 DAS, bispyribac $25 \mathrm{~g} \mathrm{ha}^{-1}+$ azimsulfuron $30 \mathrm{~g} \mathrm{ha}^{-1}$ at 15 DAS during averaged 2 years (Table 3). Our results support the findings of Brar and Bhullar (2013) that the weeds in herbicidal plot removed significantly higher amount of nitrogen, phosphorous and potassium as compared to three hand weedings. The nutrient removal by weeds was directly related to the weed dry matter accumulation under different treatments. Amongst the

Table 2: Effect of integrated weed management on soil organic carbon (\%), pH, EC, available nutrients in direct seeded rice (average of 2 years).

\begin{tabular}{|c|c|c|c|c|c|c|}
\hline Treatment & $\begin{array}{c}\text { Organic } \\
\text { carbon }(\%)\end{array}$ & $\begin{array}{l}\text { Soil } \\
\mathrm{pH}\end{array}$ & EC & $\begin{array}{l}\text { Available } \\
\mathrm{N}\left(\mathrm{kg} \mathrm{ha}^{-1}\right)\end{array}$ & $\begin{array}{l}\text { Available } \\
\mathrm{P}\left(\mathrm{kg} \mathrm{ha}^{-1}\right)\end{array}$ & $\begin{array}{l}\text { Available } \\
\mathrm{K}\left(\mathrm{kg} \mathrm{ha}^{-1}\right)\end{array}$ \\
\hline $\begin{array}{l}\mathrm{T}_{1} \text { : Sesbania cover crop fb bispyribac } 25 \mathrm{~g} \mathrm{ha}^{-1}+ \\
\text { azimsulfuron } 30 \mathrm{~g} \mathrm{ha}^{-1} \text { at } 15 \text { DAS }\end{array}$ & 0.40 & 7.45 & 0.21 & 173.83 & 18.87 & 188.08 \\
\hline $\begin{array}{l}\mathrm{T}_{2}: \text { Sunhemp cover crop fb bispyribac } 25 \mathrm{~g} \mathrm{ha}^{-1}+ \\
\text { azimsulfuron } 30 \mathrm{~g} \mathrm{ha}^{-1} \text { at } 15 \text { DAS }\end{array}$ & 0.40 & 7.44 & 0.21 & 173.00 & 18.85 & 187.57 \\
\hline $\begin{array}{l}\mathrm{T}_{3}: \text { Sesbania cover crop fb Sesbania coculture } \\
\quad f b 2,4 \mathrm{D} 0.5 \mathrm{~kg} \mathrm{ha}^{-1} \text { at } 30 \mathrm{DAS}\end{array}$ & 0.39 & 7.43 & 0.21 & 171.67 & 18.81 & 187.19 \\
\hline $\begin{array}{l}\mathrm{T}_{4}: \text { Sunhemp cover crop fb Sunhemp coculture } \\
\quad f b 2,4 \mathrm{D} 0.5 \mathrm{~kg} \mathrm{ha}^{-1} \text { at } 30 \text { DAS }\end{array}$ & 0.39 & 7.42 & 0.21 & 171.17 & 18.78 & 187.01 \\
\hline $\mathrm{T}_{5}:$ Sesbania coculture $\mathrm{fb} 2,4$-D $0.5 \mathrm{k} \mathrm{gha}^{-1}$ at 30 DAS & 0.39 & 7.41 & 0.21 & 170.33 & 18.77 & 186.83 \\
\hline $\mathrm{T}_{6}:$ Sunhemp coculture $f b 2,4-\mathrm{D} 0 . \mathrm{kg} \mathrm{ha}^{-1}$ at $30 \mathrm{DAS}$ & 0.39 & 7.41 & 0.21 & 169.00 & 18.74 & 186.65 \\
\hline $\mathrm{T}_{7}$ : Bispyribac $25 \mathrm{~g} \mathrm{ha}^{-1}+$ azimsulfuron $30 \mathrm{~g} \mathrm{ha}^{-1}$ at $15 \mathrm{DAS}$ & 0.39 & 7.44 & 0.21 & 172.00 & 18.84 & 187.54 \\
\hline $\mathrm{T}_{8}:$ Hand weeding at 15 and 35 DAS & 0.39 & 7.43 & 0.22 & 170.67 & 18.65 & 185.83 \\
\hline $\mathrm{T}_{9}:$ Weedy & 0.38 & 7.41 & 0.21 & 176.17 & 19.27 & 189.17 \\
\hline $\mathrm{SEm} \pm$ & 0.00 & 0.00 & 0.00 & 0.23 & 0.01 & 0.14 \\
\hline CD (5\%) & 0.00 & 0.01 & 0.00 & $0.68^{*}$ & 0.02 & $0.43^{*}$ \\
\hline
\end{tabular}

*Significant at $\mathrm{P} \leq 0.05$; NS- Non significant at $\mathrm{P}>0.05$.

Table 3: Effect of integrated weed management on $\mathrm{N}, \mathrm{P}, \mathrm{K}\left(\mathrm{kg} \mathrm{ha}^{-1}\right)$ and $\mathrm{Zn}\left(\mathrm{g} \mathrm{ha}^{-1}\right)$ depletion by weeds and uptake by crop at harvest in direct seeded rice (average of 2 years).

\begin{tabular}{|c|c|c|c|c|c|c|c|c|}
\hline \multirow{2}{*}{ Treatment } & \multicolumn{4}{|c|}{$\begin{array}{l}\text { Nutrient depletion by } \\
\text { weeds at harvest }\end{array}$} & \multicolumn{4}{|c|}{$\begin{array}{l}\text { Nutrient uptake by } \\
\text { crop at harvest }\end{array}$} \\
\hline & $\begin{array}{c}\mathrm{N} \\
\left(\mathrm{kg} \mathrm{ha}^{-1}\right)\end{array}$ & $\begin{array}{c}\mathrm{P} \\
\left(\mathrm{kg} \mathrm{ha}^{-1}\right)\end{array}$ & $\begin{array}{c}\mathrm{K} \\
\left(\mathrm{kg} \mathrm{ha}{ }^{-1}\right)\end{array}$ & $\begin{array}{l}\mathrm{Zn} \\
\left(\mathrm{g} \mathrm{ha} \mathrm{h}^{-1}\right)\end{array}$ & $\begin{array}{c}\mathrm{N} \\
\left(\mathrm{kg} \mathrm{ha}^{-1}\right)\end{array}$ & $\begin{array}{c}\mathrm{P} \\
\left(\mathrm{kg} \mathrm{ha}^{-1}\right)\end{array}$ & $\begin{array}{c}\mathrm{K} \\
\left.(\mathrm{kg} \mathrm{ha})^{-1}\right)\end{array}$ & $\begin{array}{c}\mathrm{Zn} \\
\left(\mathrm{g} \mathrm{ha} \mathrm{h}^{-1}\right)\end{array}$ \\
\hline $\begin{aligned} \mathrm{T}_{1} & \text { : Sesbania cover crop fb bispyribac } 25 \mathrm{~g} \mathrm{ha}^{-1}+ \\
& \text { azimsulfuron } 30 \mathrm{~g} \mathrm{ha}^{-1} \text { at } 15 \text { DAS }\end{aligned}$ & 0.7 & 0.4 & 0.8 & 28.3 & 76.8 & 21.2 & 113.4 & 3997.1 \\
\hline $\begin{aligned} \mathrm{T}_{2} & \text { : Sunhemp cover crop fb bispyribac } 25 \mathrm{~g} \mathrm{ha}^{-1}+ \\
& \text { azimsulfuron } 30 \mathrm{~g} \mathrm{ha}^{-1} \text { at } 15 \text { DAS }\end{aligned}$ & 0.9 & 0.5 & 1.0 & 30.3 & 74.2 & 19.7 & 108.2 & 3829.1 \\
\hline $\begin{array}{l}\mathrm{T}_{3}: \text { Sesbania cover crop fb Sesbania coculture } \\
\quad f b 2,4 \text { D } 0.5 \mathrm{~kg} \mathrm{ha}^{-1} \text { at } 30 \text { DAS }\end{array}$ & 1.2 & 0.7 & 1.3 & 37.4 & 70.8 & 17.8 & 104.8 & 3704.6 \\
\hline $\begin{array}{l}\mathrm{T}_{4}: \text { Sunhemp cover crop fb Sunhemp coculture } \\
\quad f b 2,4 \mathrm{D} 0.5 \mathrm{~kg} \mathrm{ha}^{-1} \text { at } 30 \mathrm{DAS}\end{array}$ & 1.3 & 0.8 & 1.4 & 39.9 & 69.3 & 16.8 & 103.1 & 3640.8 \\
\hline $\mathrm{T}_{5}$ : Sesbania coculture $\mathrm{fb} 2,4$-D $0.5 \mathrm{~kg} \mathrm{ha}^{-1}$ at 30 DAS & 1.4 & 0.8 & 1.5 & 41.8 & 67.5 & 15.7 & 100.5 & 3530.8 \\
\hline $\mathrm{T}_{6}:$ Sunhemp coculture $f b 2,4-\mathrm{D} 0.5 \mathrm{~kg} \mathrm{ha}^{-1}$ at $30 \mathrm{DAS}$ & 1.5 & 0.9 & 1.6 & 44.0 & 65.8 & 15.1 & 98.0 & 3427.1 \\
\hline $\mathrm{T}_{7}$ : Bispyribac $25 \mathrm{~g} \mathrm{ha}^{-1}+$ azimsulfuron $30 \mathrm{gha}^{-1}$ at $15 \mathrm{DAS}$ & 1.1 & 0.6 & 1.1 & 34.2 & 72.6 & 19.1 & 107.2 & 3793.0 \\
\hline $\mathrm{T}_{8}:$ Hand weeding at 15 and 35 DAS & 0.2 & 0.2 & 0.2 & 16.4 & 92.4 & 27.3 & 139.2 & 5037.8 \\
\hline $\mathrm{T}_{9}:$ Weedy & 8.1 & 3.7 & 9.5 & 158.0 & 38.0 & 7.8 & 58.1 & 1761.0 \\
\hline SEm \pm & 0.04 & 0.03 & 0.05 & 1.12 & 1.68 & 0.42 & 3.16 & 91.57 \\
\hline CD (5\%) & $0.12^{*}$ & $0.08^{*}$ & $0.14^{*}$ & $3.36^{*}$ & $5.05^{*}$ & $1.25^{*}$ & $9.48^{*}$ & $274.54^{*}$ \\
\hline
\end{tabular}

*Significant at $\mathrm{P} \leq 0.05$; NS- Non significant at $\mathrm{P}>0.05$. 
integrated weed management treatments, Sesbania cover crop fb bispyribac $25 \mathrm{~g} \mathrm{ha}^{-1}+$ azimsulfuron $30 \mathrm{~g} \mathrm{ha}^{-1}$ at 15 DAS recorded higher nutrient content while minimum depletion of nutrients by weeds as compared to all other integrated weed management treatments except hand weeding at 15 and 35 DAS. Sesbania cover crop fb bispyribac $25 \mathrm{~g} \mathrm{ha}^{-1}+$ azimsulfuron $30 \mathrm{~g} \mathrm{ha}^{-1}$ at 15 DAS reduced nutrients depletion by weeds as weed infestation and their dry matter accumulation in these treatments were statistically lower than all other integrated weed management treatments except hand weeding at 15 and 35 DAS. Similar findings reported by Sanodiya and Singh (2018) that weed management practices in DSR reduced the nutrient depletion by weeds. Amongst the integrated weed management treatments, the highest $\mathrm{N}, \mathrm{P}, \mathrm{K}$ and $\mathrm{Zn}$ depletion by weeds was recorded under sunhemp coculture $\mathrm{fb} 2,4-\mathrm{D} 0.5 \mathrm{~kg} \mathrm{ha}^{-1}$ at $30 \mathrm{DAS}$. It was due to higher weed dry matter under this treatment as compared to rest of the integrated weed management treatments except weedy and nutrient depletion is known to be positively correlated with weed dry matter accumulation. This is in agreement with the findings of Singh et al., (2014).

\section{Nutrient uptake by crop}

Mean data of 2 years showed that all integrated weed management treatments brought significant variation in nutrient uptake by rice compared with weedy (Table 3). Hand weeding at 15 and 35 DAS resulted in the highest nutrients (NPKZn) uptake by crop.Sesbania cover crop fb bispyribac $25 \mathrm{~g} \mathrm{ha}^{-1}+$ azimsulfuron $30 \mathrm{~g} \mathrm{ha}^{-1}$ at 15 DAS recorded significantly higher nutrients (NPKZn) uptakein comparison to sunhemp cover crop fb bispyribac $25 \mathrm{~g} \mathrm{ha}^{-1}+$ azimsulfuron $30 \mathrm{~g} \mathrm{ha}^{-1}$ at 15 DAS, Sesbania cover crop fb Sesbania coculture $\mathrm{fb} 2,4 \mathrm{D} 0.5 \mathrm{~kg} \mathrm{ha}^{-1}$ at 30 DAS, sunhemp cover crop fb sunhemp coculture fb 2, 4 D $0.5 \mathrm{~kg} \mathrm{ha}^{-1}$ at 30 DAS, Sesbania coculture fb 2, 4 -D $0.5 \mathrm{~kg} \mathrm{ha}^{-1}$ at $30 \mathrm{DAS}$, sunhemp coculture $\mathrm{fb} 2,4-\mathrm{D} 0.5 \mathrm{~kg} \mathrm{ha}^{-1}$ at 30 DAS, bispyribac $25 \mathrm{~g}$ $\mathrm{ha}^{-1}+$ azimsulfuron $30 \mathrm{~g} \mathrm{ha}^{-1}$ at 15 DAS. Sanodiya and Singh (2018) also showed that application of herbicides controlled weeds effectively and made available more nutrients to rice crop and consequently resulted in higher yield in direct seeded rice. At harvest stage, amongst the integrated weed management treatments, Sesbania cover crop fb bispyribac $25 \mathrm{~g} \mathrm{ha}^{-1}+$ azimsulfuron $30 \mathrm{~g} \mathrm{ha}^{-1}$ at 15 DAS recorded higher nutrient content and uptake by grain and straw as compared to all other integrated weed management treatments except hand weeding at 15 and 35 DAS. Amongst the integrated weed management treatments, the highest total $\mathrm{N}, \mathrm{P}, \mathrm{K}$ and $\mathrm{Zn}$ uptake was observed under Sesbania cover crop fb bispyribac $25 \mathrm{~g} \mathrm{ha}^{-1}+$ azimsulfuron $30 \mathrm{~g} \mathrm{ha}^{-1}$ at 15 DAS except hand weeding at 15 and 35 DAS. This is owing to lower nutrient depletion by weeds under these treatments (Table 3), whereas the lowest uptake of nutrients (N, P, K and $\mathrm{Zn}$ ) by crop was recorded under weedy. Hence, it appears that competition for nutrients was more serious due to luxuriant weed growth in weedy which resulted in less availability of nutrient and uptake by crop. The results are in close proximity with findings of Sanodiya and Singh (2018) reported that $\mathrm{N}, \mathrm{P}$ and $\mathrm{K}$ uptake by rice crop was inversely proportional to the $\mathrm{N}, \mathrm{P}$ and $\mathrm{K}$ depletion by weeds. The highest N, P and $\mathrm{K}$ uptake by crop was observed in weed free plots. Sanodiya and Singh (2018) also suggested that weed control helped in better utilization of nutrients and was recorded to be the highest in weed free plots.

\section{CONCLUSION}

On basis of two years investigation it may be concluded that Sesbania cover crop fb bispyribac Na $25 \mathrm{~g} \mathrm{ha}^{-1}+$ azimsulfuron $30 \mathrm{~g} \mathrm{ha}^{-1}$ at 15 DAS should be undertaken for improved plant height, dry matter accumulation, LAI, chlorophyll content. Consequently this treatment reported lesser nutrients (NPK and Zn) depletion by weeds and higher nutrients (NPK and $\mathrm{Zn}$ ) uptake by rice at harvest besides higher grain and straw yields in direct seeded rice.

\section{REFERENCES}

Black, C.A., Evans, D.D., White, J.E., Ensminger, L.E. and Clark, F.E. (1965). Methods of soil analysis, Part I-Physical and microbiological properties, including statistics of measurement and sampling. American Society of Agronomy. pp, 677.

Brar, H.S. and Bhullar, M.S. (2013). Nutrient uptake by direct seeded rice and associated weeds as influenced by sowing date, variety and weed control. Indian Journal of Agricultural Research. 47(4): 353-358.

Chongtham, S.K., Singh, R.P. and Singh, R.K. (2016). Effect of crop establishment methods and weed management practices on weed flora and yield of direct seeded rice (Oryza sativa). Indian Journal of Agronomy. 61(1): 33-36.

Den Hollander, N.G., Bastiaans, L. and Kropff, M.J. (2007a). Clover as a cover crop for weed suppression in an intercropping design, Characteristics of several clovers species. European Journal of Agronomy. 26: 92-103.

Gupta, R., Jat, M.L., Singh, S., Singh, V.P. and Sharma, R.K. (2006). Resource conservation technologies for rice production. Indian Farming. 56(7): 42-45.

Kruidhof, H., Bastiaans, M.L. and Kropff, M.J. (2008). Ecological weed management by cover cropping: Effects on weed growth in autumn and weed establishment in spring. Weed Research. 48: 492-502.

Kruidhof, H.M., Bastiaans, L. and Kropff, M.J. (2008b). Cover crop residue management for optimizing weed control. Plant Soil. doi: 10.1007/s11104-008-9827-6.

Kumar, J., Singh, D., Puniya, R. and Pandey, P.C. (2010). Effect of weed management practices on nutrient uptake by direct seeded rice. Oryza. 47: 291-94.

Liebman, M. and Davis, A.S. (2000). Integration of soil, crop and weed management in low-external-input farming systems. Weed Research. 40: 27-47.

Maity, S.K. and Mukherjee, P.K. (2009). Effect of brown manuring on grain yield and nutrient use efficiency in dry direct seeded Kharif rice (Oryza sativa L.). Indian Journal of Weed Science. 43(1 and 2): 61-66.

Majhi, S., Thakur, R., Upasani, R.R., Singh, M.K. and Pal, S.K. (2009). Growth and productivity of direct seeded upland rainfed rice as influenced by integrated weed management. Indian Journal of Weed Science. 41(3 and 4): 160-162. 
Malviya, A. and Singh, B. (2007). Weed dynamics, productivity and economics of maize as affected by integrated weed management under rainfed condition. Indian Journal of Agronomy. 52(4): 321-24.

Menon, S.S. and Prameela, P. (2015). Promising post emergence herbicides for effective weed management in direct seeded rice. International Journal of Tropical Agriculture. 33(2): 327-331.

Munivappa, T.V., Shetty, S.V.R., Krishnamurthy, K. and Shwaraj, B. (1980). Fertilizer use efficiency in paddy in relation to weed control through herbicides. Abstract of papers Indian Society of Weed Science Bhubaneshwar Orissa.

Nandlal, D.P. and Singh, C.M. (1995). Effect of herbicides on weed control and nutrient uptake in direct seeded puddled rice. Annals of Agricultural Research. 16: 12-15.

Nanjappa, H.V. and Krishnamurthy, K. (1980). Nutrient losses due to weed competition in tall and dwarf varities of rice. Indian Journal of Agronomy. 25(2): 273-278.

Payman, G. and Singh, S. (2008). Effect of seed rate, spacing and herbicide use on weed management in direct seeded upland rice (Oryza sativaL.). Indian Journal of Weed Science. 40(1 and 2): 11-15.

Raj, S.K., Jose, N., Mathew, R. and Leenakumary, S. (2013). Chemical management of non-grassy weeds in direct-seeded rice. Indian Journal of Weed Science. 45(3): 159-162.

Reader, R.J. (1991). Control of seedlings emergence by ground cover a potential mechanism involving seed predation. Canadian Journal of Botany. 69: 2084-2087.

Sanodiya, P. and Singh, M.K. (2017). Impact of live mulches, cover crops and herbicides on weeds, growth attributes and yield of direct seeded rice (Oryza sativa L.). Journal of Pharmacognosy and Phytochemistry. SP1: 962-966.
Sanodiya, P. and Singh, M.K. (2018). Effect of live mulches, cover crops and herbicides on weed flora, growth and yield of direct-seeded rice (Oryza sativa). Indian Journal of Agronomy. 63(1): 60-66.

Sanodiya, P. and Singh, M.K. (2018). Impact of live mulches, cover crops and herbicides on weeds and yield of direct-seeded rice. Indian Journal of Weed Science. 50(2): 112-115.

Sanodiya, P. and Singh, M.K. (2019). Impact of integrated weed management on weeds and yield of direct-seeded rice (Oryza sativa). Indian Journal of Agronomy. 64(1): 64-68.

Sharma, R.P., Pathal, S.K. and Singh, R.C. (2007). Effect of nitrogen and weed management in direct-seeded rice (Oryza sativa) under upland conditions. Indian Journal of Agronomy. 52(2): 114-19.

Singh, R., Pal, R., Singh, T., Singh, A.P., Yadaw, S. and Singh, J. (2014). Management of weeds in direct-seeded rice by bispyribac-sodium. Indian Journal of Weed Sciences. 46(2): 126-128.

Singh, S., Bhushan, L., Ladha, J.K., Gupta, R.K., Rao, A.N. and Sivaprasad, B. (2006). Weed management in dry-seeded rice (Oryza sativa L.) cultivated on furrow irrigated raised bed planting system. Crop Protection. 25: 487-495.

Sunil, C.M., Shekara, B.G., Kalyanamurthy, K.N. and Shankaralingappa, B.C. (2010). Growth and yield of aerobic rice as influenced by integrated weed management practices. Indian Journal of Weed Science. 42(3 and 4): 180-183.

Teasdale, J.R. and Daughty, C.S.T. (1993). Weed suppression by live and desiccated hairy vetch. Weed Science. 41: 207212. 